\title{
Synthesis and microwave absorption studies of ferrite paint ${ }^{\$}$
}

\author{
S M ABBAS, R P R C AIYAR ${ }^{\dagger}$ and OM PRAKASH* \\ Department of Metallurgical Engineering and Materials Science, 'ACRE, Indian Institute of Technology, \\ Mumbai 400 076, India
}

MS received 19 December 1997; revised 17 March 1998

\begin{abstract}
A new hexaferrite composition, $\mathrm{BaCo}_{x} \mathrm{Si}_{x+y} \mathrm{Fe}_{12-2 x-y} \mathrm{O}_{19}(x=0.90 ; y=0.05)$, in fine $(\sim \mu$ size $)$ powder form, is prepared. Ferrite paint formulations are made by dispersing this powder in different weight proportions in an epoxy resin. X-band (8-12 GHz) microwave absorption behaviour of the paint coatings of different thicknesses is studied. Remarkably high value, $\geq 10 \mathrm{~dB}$, of signal absorption at $9.5 \mathrm{GHz}$ is observed for a coating thickness of $0.60 \mathrm{~mm}$ with a ferrite fill factor of $57 \mathrm{wt} \%$.
\end{abstract}

Keywords. Hexaferrite; microwave absorption; ferrite paint.

\section{Introduction}

$M$-type hexaferrites, with large B-H product, are widely used as permanent magnets. Because of their high values of magnetization $\left(M_{s}\right)$ and intrinsic anisotropy $(K)$, hexaferrites are important for microwave applications. The hexaferrite also finds application as absorber for microwaves (Naito and Suetake 1971; Amin and James 1981). A coating of ferrite paint on a microwave reflecting surface can significantly attenuate (absorb) the incident signals (Aiyar et al 1989). Consequently, an aeroplane, with such a coating, can escape, to an extent, its detection by radar (Goldman 1990). The microwave absorbing paints therefore play an important role in stealth technology (John 1988) and ghost signal attenuation e.g. ship-to-ship microwave communication (Mohammed 1989). Ferrite paints, because of their strategic applications, are patented in nature and therefore the formulation details are rarely available in the published literature.

Fine $\left(\mu\right.$ size) powders of hexaferrite e.g. $\mathrm{BaTi}_{\delta} \mathrm{Co}_{\delta}-$ $\mathrm{Fe}_{12-2 \delta} \mathrm{O}_{19}, 0<\delta<1.5$ (Amin and James 1981; Aiyar et al 1989), are the major constituents of a typical microwave absorbing paint formulation, while epoxy resin and graphite powder are the minor constituents. These ferrites, due to their large intrinsic anisotropy field, $H_{\mathrm{A}}$ $\left(=2 K / M_{\mathrm{s}}\right)$, cause the natural magnetic resonance frequency $\left(f_{0}\right)$, where absorption is maximum, to fall in the microwave region $\left(f_{1}=\gamma H_{\mathrm{A}}\right.$, where $\gamma$ is gyromagnetic ratio). Further, $f_{0}$ can be tuned to any desired frequency region between $2-52 \mathrm{GHz}$ by engineering the anisotropy

\footnotetext{
${ }^{\text {Most of this work, in a poster form, was presented at the } 8 \text { th }}$ AGM of MRSI, BARC, Mumbai, February 10-12, 1997. The paper was adjudged the best for poster paper award

*Author for correspondence
}

field, $H_{\mathrm{A}}$, through appropriate cationic substitution for some of the $\mathrm{Fe}^{3+}$ ions in $\mathrm{BaFe}_{12} \mathrm{O}_{19}$ (Severin and Stoll 1967).

The ferrite paint formulations reported here are made of a single composition $\mathrm{Co}-\mathrm{Si}$ substituted Ba-hexaferrite fine powder as pigment, and epoxy resin as a matrix. The choice of $\mathrm{Si}^{4+}$ ion substitution is made to reduce a part of $\mathrm{Fe}^{3+}$ to $\mathrm{Fe}^{2+}$. This, due to electron hopping among $\mathrm{Fe}^{2+}$ and $\mathrm{Fe}^{3+}$ ions, reduces the electrical resistivity and makes the hexaferrite material more lossy at microwave frequencies. The dependence of microwave absorption characteristics on the fill factor of ferrite and the thickness of the paint coatings has been studied. The details of such a study are presented here.

\section{Experimental}

\subsection{Synthesis of hexaferrite powder}

The ferrite samples of composition $\mathrm{BaCo}_{x} \mathrm{Si}_{x+y} \mathrm{Fe}_{12-2 x-y} \mathrm{O}_{19}$ $(x=0.90 ; y=0.05)$ were synthesized by solid state reaction route. The starting raw materials were $\mathrm{BaCO}_{3}, \mathrm{Fe}_{2} \mathrm{O}_{3}$, $\mathrm{SiO}_{2}$ and $\mathrm{Co}_{3} \mathrm{O}_{4}$ with a purity $\geq 98 \%$. The ingredients were mixed in stoichiometric proportion, and finely ground in acetone medium in a laboratory planetary mill. The finely mixed powder was compacted at $20 \mathrm{MPa}$ into pellets and calcined in air at $-960^{\circ} \mathrm{C}$ for $5 \mathrm{~h}$. The calcined pellets, after crushing, regrinding and repelletization (at $50 \mathrm{MPa}$ ) were sintered at $\sim 1150^{\circ} \mathrm{C}$ for $5 \mathrm{~h}$ in air and furnace cooled. The low sintering temperature was selected to ensure complete phase formation and avoid the temperature-assisted grain growth. The sintered pellets were finely ground to obtain $\mu$ size ferrite powder.

\section{$2.2 X$-ray diffraction}

The sintered samples in powder form were analysed for phase formation and phase purity by $\mathrm{X}$-ray diffraction 
pattern, using a Philips X-ray diffractometer (Model PW $1820)$, and $\mathrm{Cu}-\mathrm{K}_{\alpha}$ radiation of wavelength $1.542 \AA$.

\subsection{Particle size analysis}

Particle size distribution for the ferrite powder was obtained by a He-Ne-laser-based particle analyzer (GalaiCIS-1, version 4.2). Following this method, a laser beam is focused and narrowed down to $1.0 \mu \mathrm{m}$ spot; as the beam rotates at a constant speed, the powder particles in a specified sample volume are individually bisected by the laser spot and interaction signals are generated. The duration of interaction provides a direct measurement of each particle volume.

\subsection{Scanning electron microscope}

Morphology of the ferrite powder was studied by scanning electron microscope (SEM) (model JSM 840A, JEOL). Highly polished brass plates were used for placing the powder particles for measurements. Powder was agitated ultrasonically in acetone and a drop was put on the polished substrate and allowed to dry rapidly.

\subsection{Paint formulation and thick film formation}

The ferrite powder was used as a pigment, and an epoxy Novolac resin, a product of phenol and formaldehyde (for more details, see Raju 1997), was used as a binder medium. Resin was cured by diethylamine (as curing agent). The base resin and the curing agent were added in equal proportion by volume. Different volume $\%$ of the ferrite powder were poured into measured volume

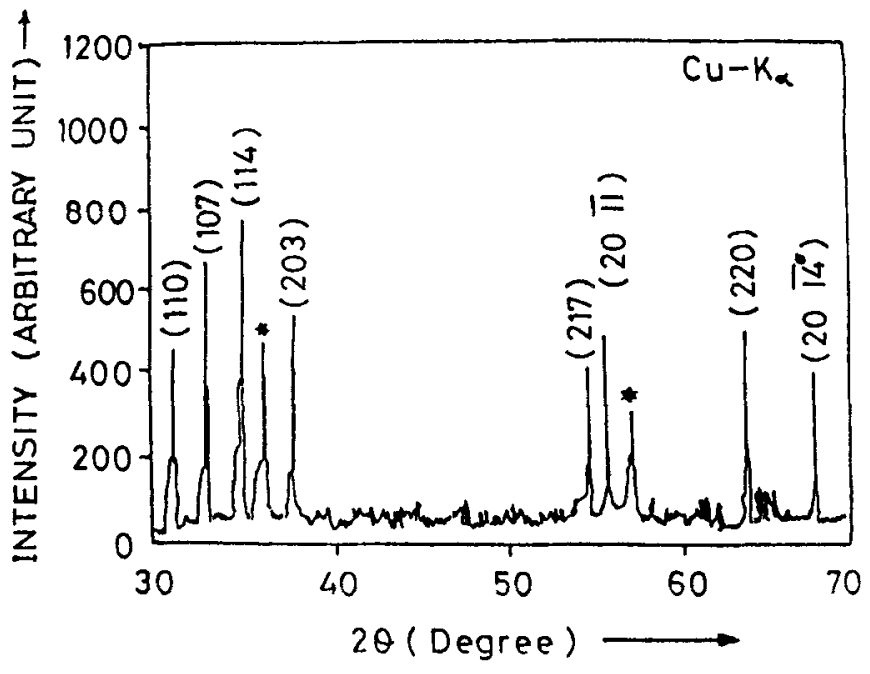

Figure 1. Powder XRD pattern of hexaferrite sample of composition $\mathrm{BaCo}_{x} \mathrm{Si}_{x+y} \mathrm{Fe}_{12-2 x-y} \mathrm{O}_{19}(x=0.90 ; y=0.06)$ sintered at $1150^{\circ} \mathrm{C}$. Peaks marked $*$ are due to unreacted $\alpha-\mathrm{Fe}_{2} \mathrm{O}_{3}$. of the resin. Further, around $40 \%$ by volume, acetone was added to reduce the viscosity of the resin. Each mixture was stirred and simultaneously agitated ultrasonically to have a homogeneous, well-dispersed ferriteepoxy paint.

The freshly prepared paint was immediately applied on to the surface of an aluminium substrate of specified surface dimensions $(10 \mathrm{~mm} \times 22.5 \mathrm{~mm})$ required for $\mathrm{X}$ band ( $8-12 \mathrm{GHz})$ microwave absorption studies. The thick film was allowed to cure overnight in air. Cured and dried films were trimmed to fit into the X-band waveguide. A large number of such coatings with different ferrite contents and of varying thicknesses were formed for the absorption studies.

\subsection{Microwave absorption measurements}

The ferrite paint coatings were tested for their absorption properties by employing the Wiltron network analyzer (model 360B) in the frequency range of $8-12 \mathrm{GHz}$. The network analyzer was calibrated using standard one port calibration procedure. The reflection coefficient of the paint coated substrate was found by measuring the $S$ parameter $\left(S_{11}\right)$. In order to determine the reflection coefficient of coating alone, the $S$-parameter of the uncoated substrate was used as a reference.

\section{Results and discussion}

The powder XRD pattern for the composition $\mathrm{BaCo}_{x} \mathrm{Si}_{x+y} \mathrm{Fe}_{12-2 x-y} \mathrm{O}_{19} \quad(x=0.90 ; y=0.05)$, shown in figure 1 , reveals formation of the $M$-type hexaferrite phase. In addition, a couple of lines of considerable intensity due to $\alpha-\mathrm{Fe}_{2} \mathrm{O}_{3}$ and a few weak lines due to

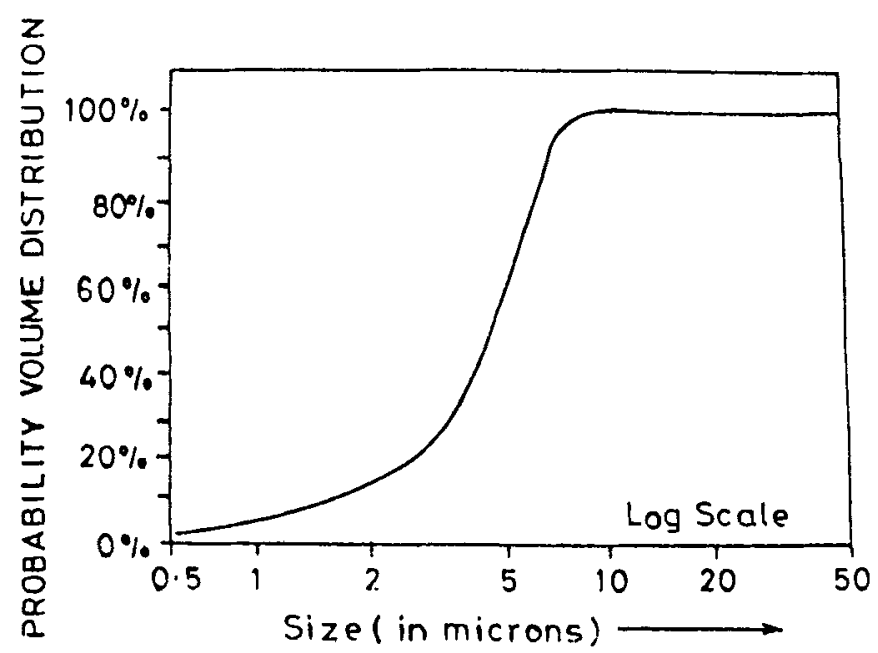

Figure 2. Particle size (volume) distribution of hexaferrite powder used in paint formulation. 
unidentifiable trace impurities are also present. The impurity phases nevertheless add to the microwave losses as a dielectric rather than a magnetic medium in the paint. Figure 2 shows the cumulative particle size (volume) distribution of the ferrite powder used for paint formulation, and gives no indication of wide variation in the particle size. The SEM micrograph of the same ferrite powder (figure 3 ), reveals a narrow size distribution (micron range) of irregularly-shaped particles.

$X$-band microwave absorption spectra obtained for the hexaferrite-epoxy paint coated substrates are shown in figure 4 . The fill factor for the probe sample was $57 \%$ by weight of ferrite content. It is observed that the peak absorption increases, in general, with increasing thickness of the paint coating, and somewhat shifts towards lower side of the frequency band (figure 4). A maximum absorption of $6 \mathrm{~dB}$ with a bandwidth of $0.6 \mathrm{GHz}$ was found for single layer of thickness $1.2 \mathrm{~mm}$. Absorption was also measured on another sample of $\sim 0.6 \mathrm{~mm}$ thickness of similar composition and fill factor. Interestingly, this showed a peak absorption of more than $10 \mathrm{~dB}$ albeit with a very narrow bandwidth (figure 5). This increase in absorption at lower thickness, apparently showing anomaly to what is stated earlier, can possibly be explained by using the criterion of a good absorber, as given by Naito and Suetake (1971). In their analysis, the reflection and transmission at the interface between the free space and the ferrite medium (backed by a perfect metallic wall) is considered for normal incidence of an electromagnetic wave. It is shown that the condition for perfect matching corresponds to the case when impedance $(Z)$ of the medium is equal to the impedance of free space $\left(Z_{0}\right)$ at the interface,

$$
Z=\left(E_{0}-E_{0} \mathrm{e}^{-2 \jmath k d}\right) /\left(H_{0}+H_{0} \mathrm{e}^{-2 k k d}\right)
$$

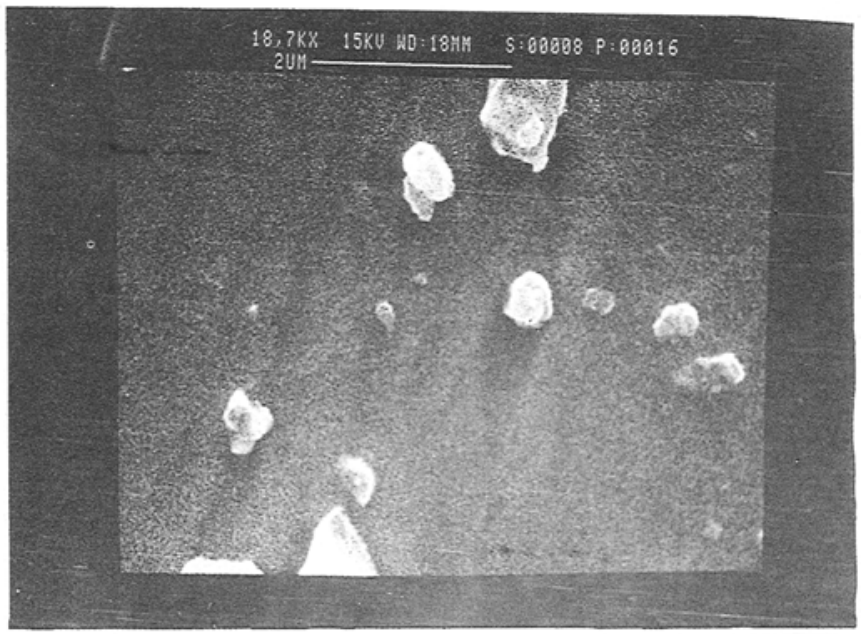

Figure 3. SEM photomicrograph of ferrite powder used in the paint. where, $E_{0}, H_{0}$ are the electric field and magnetic field amplitudes, $k$ the propagation constant in ferrite medium and $d$ the thickness of the ferrite medium. The second term in numerator and denominator of (1) arises as the wave travels a total distance of $2 d$ in the ferrite medium before coming out of the interface after reflection. This gives

$$
Z=Z_{0}\left(\mu_{\mathrm{r}} / \varepsilon_{\mathrm{r}}\right)^{1 / 2} \tan h\left[(j 2 \pi / \lambda) d\left(\mu_{\mathrm{r}} \varepsilon_{\mathrm{r}}\right)^{1 / 2}\right],
$$

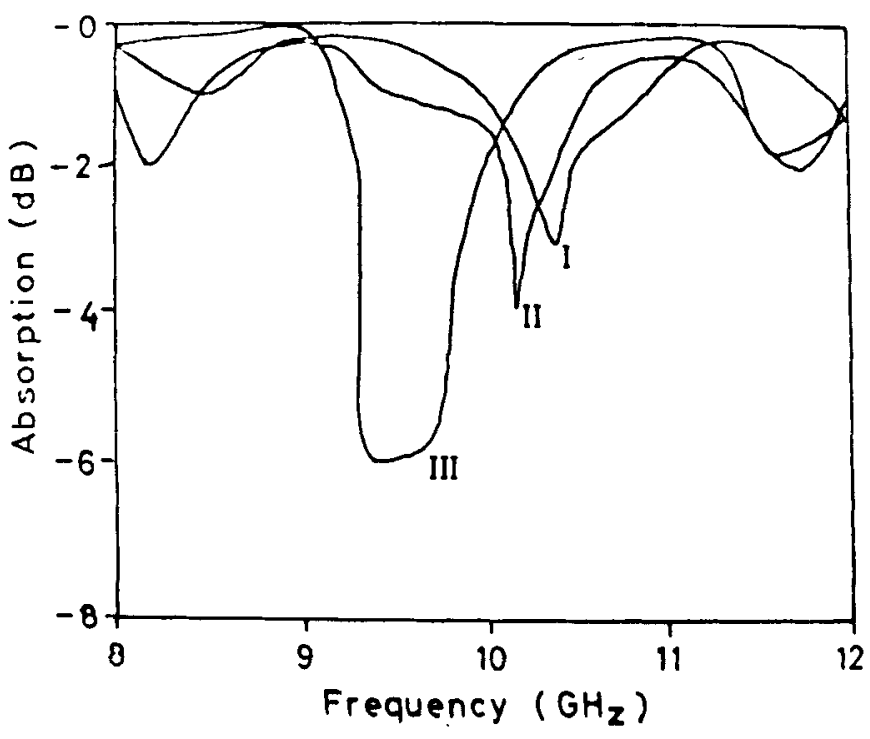

Figure 4. Microwave absorption spectra of ferrite-epoxy paint for $57 \mathrm{wt} \%$ fill factor and thickness: (I) $0.5 \mathrm{~mm}$, (II) $0.75 \mathrm{~mm}$ and (III) $1.2 \mathrm{~mm}$.

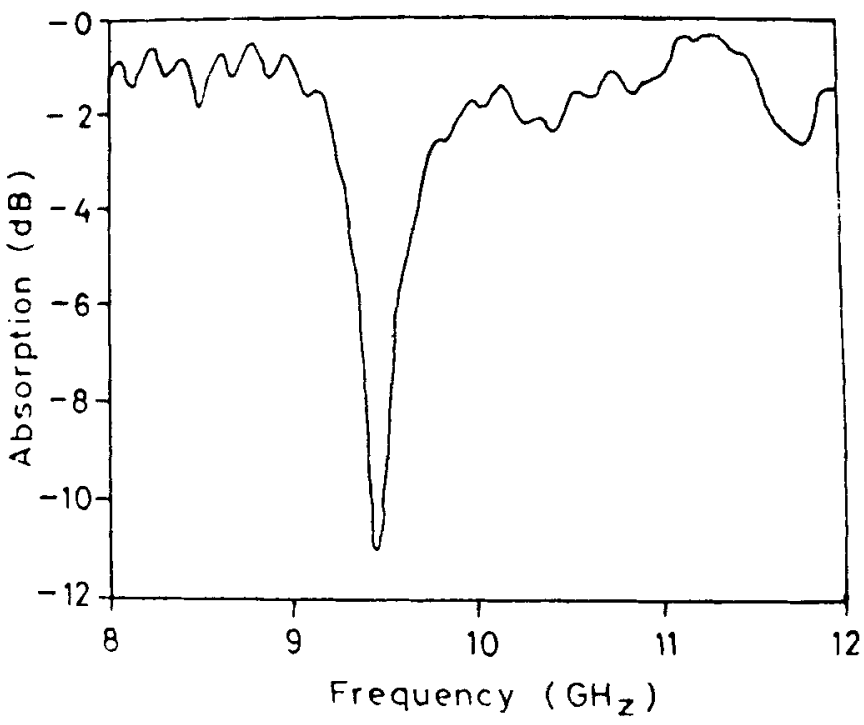

Figure 5. Microwave absorption spectrum of a second lot of ferrite-epoxy paint formulation with $\sim 57 \mathrm{wt} \%$ fill factor and $0.6 \mathrm{~mm}$ sample thickness. 
where, $\varepsilon_{r}$ and $\mu_{r}$ are the relative permittivity and permeability of the ferrite medium. For a perfect matching and absorbing medium, $Z=Z_{0}$, therefore, from (2) one gets

$$
\left(\mu_{r} \varepsilon_{r}\right)^{1 / 2} \tanh \left[(j 2 \pi / \lambda) d\left(\mu_{r} \varepsilon_{r}\right)^{1 / 2}\right]=1 .
$$

This transcendental equation gives the condition necessary for a good absorber. It also signifies the relation between the material parameters $\varepsilon_{\mathrm{r}}, \mu_{\mathrm{r}}, d$ and frequency $(f=c / \lambda)$. Since $\mu_{\mathrm{r}}$ and $\varepsilon_{\mathrm{r}}$ are frequency dependent, a quantitative interpretation of the observed results on the basis of (2) is possible only if these are determined experimentally. In the absence of measured values of $\mu_{\mathrm{r}}$ and $\varepsilon_{\mathrm{r}}$, at most a qualitative view can be given. It is quite likely that when $d=0.6 \mathrm{~mm}$, the relation (2) is satisfied, leading to a better impedance match and, consequently, a higher absorption. For the other values of $d$, the matching could be poor, leading to lower absorption. Nevertheless, this aspect needs closer investigation. Further, the measurements of complex permeability $\left(\mu^{\prime}\right.$ and $\left.\mu^{\prime \prime}\right)$, permittivity $\left(\varepsilon^{\prime}\right.$ and $\left.\varepsilon^{\prime \prime}\right)$ and the loss tangent of the coatings are expected to throw more light on the thickness parameter vis-a-vis absorption. Study on this aspect is in progress in addition to varying the chemical composition of the ferrite and the powder morphology.

\section{Conclusion}

A ferrite paint based on new hexaferrite composition is made that has successfully demonstrated microwave absorption exceeding $10 \mathrm{~dB} / \mathrm{mm}$ thickness in a narrow frequency range at $\mathrm{X}$ band.

\section{Acknowledgement}

Authors gratefully acknowledge $\mathrm{Mr}$ R S Parolia for useful discussion and help.

\section{References}

Aiyar R, Rao N S H, Uma S, Rane S A and Srivastava C M 1989 Proceedings int. conf. ferrites (ICF-5), Bombay (eds) C M Srivastava and M J Patni (New Delhi: Oxford \& IBH Publishing Co.) p. 955

Amin M B and James J R 1981 Radio Electron. Eng. 51 209

Goldman A 1990 Modern ferrite technology (New York: Van Nostrand Reinhold) p. 386

John D 1988 Aviation Week \& Space Technol. 12928

Mohammed N A 1989 Proceedings int. conf. ferrites (ICF-5), Bombay (eds) C M Srivastava and M J Patni (New Delhi: Oxford \& IBH Publishing Co.) p. 983

Naito $\mathrm{Y}$ and Suetake K 1971 IEEE Trans. Microwave Theory Tech. MTT-19 65

Raju J S 1997 Development of epoxies suitable as high temperature insulation resins, M. Tech. Thesis. IIT Bombay (unpublished)

Severin H and Stoll J P 1967 Z. Angew. Phys. 23209 